Research Article

\title{
On the Surface Fields of a Small Circular Loop Antenna Placed on Plane Stratified Earth
}

\author{
Mauro Parise \\ Faculty of Engineering, University Campus Bio-Medico of Rome, 00128 Rome, Italy \\ Correspondence should be addressed to Mauro Parise; m.parise@unicampus.it
}

Received 29 July 2015; Accepted 8 October 2015

Academic Editor: Jun $\mathrm{Hu}$

Copyright (C) 2015 Mauro Parise. This is an open access article distributed under the Creative Commons Attribution License, which permits unrestricted use, distribution, and reproduction in any medium, provided the original work is properly cited.

An analytical method is presented which makes it possible to derive exact explicit expressions for the time-harmonic surface fields excited by a small circular loop antenna placed on the top surface of plane layered earth. The developed procedure leads to casting the complete integral representations for the EM field components into forms suitable for application of Cauchy's integral formula. As a result, the surface fields are expressed as sums of Hankel functions. Numerical simulations are performed to show the validity and accuracy of the proposed solution.

\section{Introduction}

The need to evaluate the electromagnetic fields radiated by horizontal loop antennas located in close proximity to layered media arises in a variety of applications of scientific interest, especially in the areas of electromagnetics [1-9], close-tothe-surface radio communication $[10,11]$, and geophysical prospecting [11-13]. An excellent illustration of such applications is electromagnetic induction sounding technique $[12,14,15]$, which allows acquiring information about the subsurface structure of a terrestrial area. The technique consists of two steps. At first, the fields generated by a currentcarrying circular loop antenna lying on the surface of the medium to be explored are measured at a discrete set of frequencies. Next, the presence of shallow buried objects, or the geometrical and EM properties of the structure, is deduced by interpreting the recorded experimental data through matching with the theoretical response curves associated with standard homogeneous or layered earth models $[12,14,15]$. It is easily understood how, for correct data interpretation, high accuracy is required when computing such theoretical response curves.

This paper will focus on the problem of an electrically small circular loop antenna (vertical magnetic dipole) lying on the surface of $N$-layer plane stratified earth. To date, the fields of a loop source in the presence of a layered ground are not known in closed form, and evaluation of their complete integral representations has been revealed to be challenging from both analytical and numerical points of view. When available, analytical solutions either pertain to simple earth models, like the single-layer configuration [16], or follow from introducing simplifying assumptions. Examples of approximate solutions are the explicit series-form expressions that result from applying standard asymptotic techniques, such as the method of steepest descent (saddlepoint method) [11, 12, 17-19]. The obtained formulas are easy to use but are valid under the restrictive assumption that the source-receiver distance is electrically large, that is, in the far-field region of the antenna. Similar considerations can be made with regard to the low-frequency or quasi-static expressions for the fields $[7,12,13,20]$, which are accurate, subject to the condition that the displacement currents are negligible in both the layered material medium and the air space above it.

On the other hand, numerical computation of the field integrals through standard numerical approaches, like Gaussian quadrature, is often made difficult and impractical by the oscillating behavior of the integrands and the slow convergence of their envelope, especially when both source and field points lie at the air-medium interface $[1,12,21]$. 


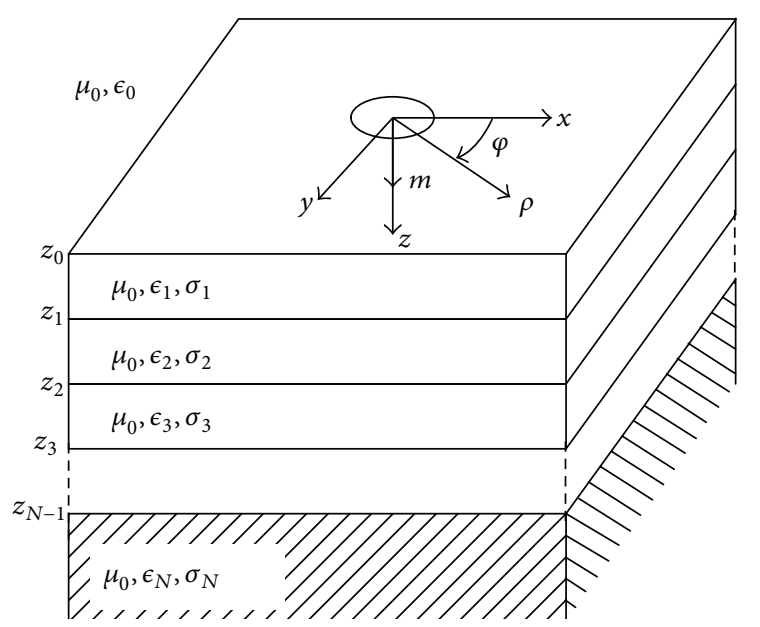

FIGURE 1: Sketch of a vertical magnetic dipole on a multilayer ground.

In an attempt to overcome these drawbacks, some papers document extensive usage of the digital linear filter technique in place of the Gaussian quadrature approach [22, 23]. Unfortunately, it has been recently shown $[9,24,25]$ that any digital filter is designed and optimized for a specific known integral transform (the so-called test function) and may lead to uncertain results when applied to the calculation of different integrals.

The aim of this work is to derive exact explicit expressions for the fields excited by the loop source at the air-medium interface. The theoretical development is organized as follows. First, the Sommerfeld Integrals describing the fields are split into two terms, that is, the residue contributions from the poles of the integrands and the branch-cut integrals due to the propagation constants in free space and in the bottom layer of the stratified medium. Next, such propagation constants are extracted from the integrands of the branch-cut integrals and replaced with the sums of partial fractions generated by Newton's iterative method [26, 27]. This makes it possible to apply Cauchy's integral formula and obtain explicit series representations for the branch-cut integrals. As a result, the Sommerfeld Integrals are converted into infinite sum of Hankel functions. The advantage of the presented approach resides in the fact that the sequence of the sums of partial fractions that are used in place of each propagation constant is convergent, as Newton's method exhibits quadratic rate of convergence if applied to the computation of a square root [26]. This implies that the derived series representations for the fields are exact.

\section{Theory}

Consider a vertical magnetic dipole of moment $m e^{j \omega t}$ lying on the surface of a flat $N$-layer lossy medium, as shown in Figure 1. Due to symmetry about the axis of the dipole, a cylindrical coordinate system $(r, \varphi, z)$ is suitably introduced. The dielectric permittivity and electric conductivity of the $n$th layer are denoted by $\epsilon_{n}$ and $\sigma_{n}$, respectively, while the medium is assumed to have the magnetic permeability of free space $\mu_{0}$. The time-harmonic EM field components generated by the dipole in the air space (the zeroth layer, $z<0$ ) may be derived from an electric vector potential which has only $z$ component $F$. If the time factor $e^{j \omega t}$ is assumed, it reads

$$
\begin{aligned}
& E_{\varphi}=\frac{\partial F}{\partial \rho}, \\
& H_{\rho}=\frac{1}{j \omega \mu_{0}} \frac{\partial^{2} F}{\partial \rho \partial z}, \\
& H_{z}=-\frac{1}{j \omega \mu_{0}}\left(\frac{\partial^{2}}{\partial \rho^{2}}+\frac{1}{\rho} \frac{\partial}{\partial \rho}\right) F,
\end{aligned}
$$

where $F=F_{d}+F_{r}$, with

$$
\begin{aligned}
& F_{d}=\frac{j \omega \mu_{0} m}{8 \pi} \int_{\infty e^{j \pi}}^{\infty} e^{u_{0} z} \frac{\lambda}{u_{0}} H_{0}^{(1)}(\lambda \rho) d \lambda, \\
& F_{r}=\frac{j \omega \mu_{0} m}{8 \pi} \int_{\infty e^{j \pi}}^{\infty} R_{\mathrm{TE}} e^{u_{0} z} \frac{\lambda}{u_{0}} H_{0}^{(1)}(\lambda \rho) d \lambda,
\end{aligned}
$$

respectively, being the direct and reflected potentials. In (2) and $(3), H_{0}^{(1)}(\cdot)$ is the zeroth-order Hankel function of the first kind, while

$$
R^{\mathrm{TE}}=\frac{Y_{0}-\widehat{Y}_{1}}{Y_{0}+\widehat{Y}_{1}}
$$

is the plane wave reflection coefficient at $z=z_{0}=0$, with $Y_{0}$ and $\widehat{Y}_{1}$ being the intrinsic admittance of free space and the surface admittance at $z_{0}$. The intrinsic admittance of the $n$th layer is given by

$$
Y_{n}=\frac{u_{n}}{j \omega \mu_{n}},
$$

with

$$
\begin{aligned}
& u_{n}=\sqrt{\lambda^{2}-k_{n}^{2}}, \quad \operatorname{Re}\left[u_{n}\right]>0, \\
& k_{n}^{2}=\omega^{2} \mu_{n} \epsilon_{n}-j \omega \mu_{n} \sigma_{n},
\end{aligned}
$$

while, for $N$ layers, the surface admittance at $z=z_{n-1}$ is obtained through the recurrence relation

$$
\begin{aligned}
\widehat{Y}_{n}=Y_{n} \frac{\widehat{Y}_{n+1}+Y_{n} \tanh \left[u_{n}\left(z_{n}-z_{n-1}\right)\right]}{Y_{n}+\widehat{Y}_{n+1} \tanh \left[u_{n}\left(z_{n}-z_{n-1}\right)\right]} & \\
n & =N-1, \ldots, 1,
\end{aligned}
$$

with

$$
\widehat{Y}_{N}=Y_{N}
$$

The reflected field may be split into two parts, that is, the ideal reflected field (the field of a negative image) and a contribution due to the imperfect conductivity of the earth. Use of the identity

$$
R^{\mathrm{TE}}=-1+\frac{2 Y_{0}}{Y_{0}+\widehat{Y}_{1}}
$$




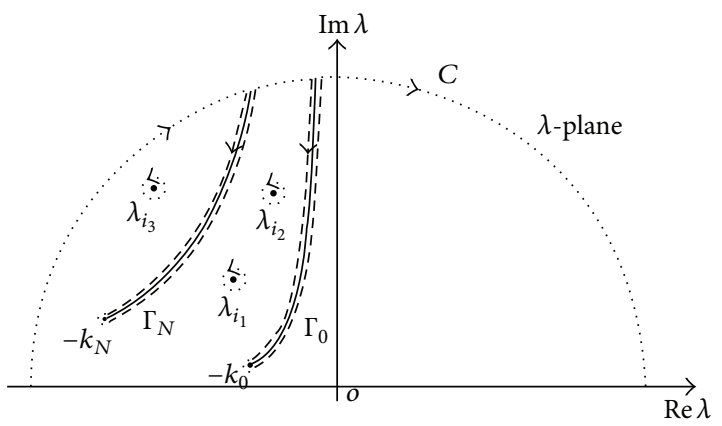

Figure 2: Deformed integration contour for the Sommerfeld Integrals.

in (3) allows concluding that the direct and image fields exactly cancel out at all points in the air space. Hence, the total potential reads

$$
\begin{aligned}
F & =\frac{j \omega \mu_{0} m}{4 \pi} \int_{\infty e^{j \pi}}^{\infty} \frac{Y_{0} e^{u_{0} z}}{Y_{0}+\widehat{Y}_{1}} \frac{\lambda}{u_{0}} H_{0}^{(1)}(\lambda \rho) d \lambda \\
& =\frac{j \omega \mu_{0} m}{4 \pi} \int_{\infty e^{j \pi}}^{\infty} \frac{e^{u_{0} z}}{u_{0}+j \omega \mu_{0} \widehat{Y}_{1}} H_{0}^{(1)}(\lambda \rho) \lambda d \lambda .
\end{aligned}
$$

The fraction under the integral sign has a number of pole singularities, plus the branch point singularities at $\lambda= \pm k_{0}$ and $\lambda= \pm k_{N}$. It is interesting to note that the points $\lambda= \pm k_{1}, \lambda= \pm k_{2}, \ldots, \lambda= \pm k_{N-1}$ are not branch points of the integrand, as the fraction term is an even function of $u_{1}, u_{2}, \ldots, u_{N-1}$. We now deform the integration path to the upper infinite semicircle $C$ centered at the origin of the complex $\lambda$-plane, leaving out all the singularities located in the first and second quadrants, as indicated in Figure 2. The result of the deformation is a new integration path constituted by $C$, the two lines wrapped around the branch cuts, that is, $\Gamma_{0}$ and $\Gamma_{N}$, and a number of infinitesimal circles around the poles of the integrand. It is not difficult to prove that the infinite semicircle does not contribute to the integral. In fact, for large values of $|\lambda|$, the Hankel function may be replaced with its asymptotic form [28]

$$
H_{0}^{(1)}(\lambda \rho) \cong \sqrt{\frac{2}{j \pi \lambda \rho}} e^{j \lambda \rho}
$$

and the integrand in (11) becomes suitable for application of Jordan's Lemma [29], which states the following upper bound:

$$
\left|\int_{C} \frac{\sqrt{\lambda} e^{u_{0} z}}{u_{0}+j \omega \mu_{0} \widehat{Y}_{1}} e^{j \lambda \rho} d \lambda\right| \leq \frac{\pi}{\rho} \max _{C}\left|\frac{\sqrt{\lambda} e^{u_{0} z}}{u_{0}+j \omega \mu_{0} \widehat{Y}_{1}}\right| .
$$

Since, in the air space, factor $e^{u_{0} z}$ decays exponentially with increasing $|\lambda|$, the right-hand side of (13) must vanish, and so does the integral along $C$. As a consequence, $F$ may be expressed as

$$
F=\frac{j \omega \mu_{0} m}{4 \pi} \int_{\Gamma} \frac{e^{u_{0} z}}{u_{0}+j \omega \mu_{0} \widehat{Y}_{1}} H_{0}^{(1)}(\lambda \rho) \lambda d \lambda,
$$

where $\Gamma$ is the contour consisting of $\Gamma_{0}, \Gamma_{N}$, and the infinitesimal circles. It should be observed that the branchcut integrals along $\Gamma_{0}$ and $\Gamma_{N}$ describe the above-surface ground wave and the lateral-wave field, respectively, while the integrals around the poles are trapped-surface-wave terms [17].

The scope of this work is to exactly evaluate the field components at the air-ground interface. To this goal, we substitute (14) into (1) and take the limit as $z \rightarrow 0^{-}$. It yields

$$
\begin{aligned}
& E_{\varphi}=\frac{j \omega \mu_{0} m}{4 \pi} \frac{\partial S_{0}}{\partial \rho}, \\
& H_{\rho}=\frac{m}{4 \pi} \frac{\partial S_{1}}{\partial \rho}, \\
& H_{z}=-\frac{m}{4 \pi}\left(\frac{\partial^{2}}{\partial \rho^{2}}+\frac{1}{\rho} \frac{\partial}{\partial \rho}\right) S_{0},
\end{aligned}
$$

with

$$
\begin{aligned}
S_{h} & =\int_{\Gamma} f_{h}(\lambda) H_{0}^{(1)}(\lambda \rho) \lambda d \lambda, \\
f_{h}(\lambda) & =\frac{u_{0}^{h}}{u_{0}+j \omega \mu_{0} \widehat{Y}_{1}} .
\end{aligned}
$$

Quantity $S_{h}$ may be rewritten as

$$
S_{h}=S_{h}^{(0)}+S_{h}^{(N)}+S_{h}^{(p)}
$$

where

$$
S_{h}^{(n)}=\int_{\Gamma_{n}} f_{h}(\lambda) H_{0}^{(1)}(\lambda \rho) \lambda d \lambda, \quad n=0, N,
$$

is the integral along $\Gamma_{n}$, while $S_{h}^{(p)}$ is the contribution from the residues of the integrand at the poles of $f_{h}$. The explicit expression for $S_{h}^{(p)}$ follows from applying Cauchy's theorem [29]. It yields

$$
\begin{aligned}
S_{h}^{(p)} & =2 \pi j \sum_{i} \Re_{i}\left[f_{h}(\lambda) H_{0}^{(1)}(\lambda \rho) \lambda\right] \\
& =2 \pi j \sum_{i} \frac{H_{0}^{(1)}\left(\lambda_{i} \rho\right) \lambda_{i}}{\left\{(d / d \lambda)\left[f_{h}(\lambda)\right]^{-1}\right\}_{\lambda=\lambda_{i}}},
\end{aligned}
$$

where $\mathfrak{R}_{i}[\cdot]$ denotes the residue at the generic pole $\lambda_{i}(i=$ $1,2, \ldots)$. On the other hand, to evaluate $S_{h}^{(0)}$ and $S_{h}^{(N)}$, it is convenient to extract the square root terms $u_{0}$ and $u_{N}$ from $f_{h}$. This task may be accomplished through letting $f_{h}(\lambda)=\phi_{h}\left(u_{0}, u_{N}\right)$ and then applying the branch-cut extraction procedure:

$$
\phi_{h}=\phi_{h}^{(e e)}+\phi_{h}^{(e o)} u_{N}+\phi_{h}^{(o e)} u_{0}+\phi_{h}^{(o o)} u_{0} u_{N}
$$


where

$$
\begin{aligned}
& \phi_{h}^{(e e)}=\frac{1}{4}\left(\phi_{h}^{++}+\phi_{h}^{-+}+\phi_{h}^{+-}+\phi_{h}^{--}\right), \\
& \phi_{h}^{(e o)}=\frac{1}{4 u_{N}}\left(\phi_{h}^{++}+\phi_{h}^{-+}-\phi_{h}^{+-}-\phi_{h}^{--}\right), \\
& \phi_{h}^{(o e)}=\frac{1}{4 u_{0}}\left(\phi_{h}^{++}-\phi_{h}^{-+}+\phi_{h}^{+-}-\phi_{h}^{--}\right), \\
& \phi_{h}^{(o o)}=\frac{1}{4 u_{0} u_{N}}\left(\phi_{h}^{++}-\phi_{h}^{-+}-\phi_{h}^{+-}+\phi_{h}^{--}\right),
\end{aligned}
$$

with

$$
\phi_{h}^{ \pm \pm}=\phi_{h}\left( \pm u_{0}, \pm u_{N}\right) .
$$

It is straightforward to verify that functions $\phi_{h}^{(e e)}, \phi_{h}^{(e o)}, \phi_{h}^{(o e)}$, and $\phi_{h}^{(o o)}$ are even in both $u_{0}$ and $u_{N}$ and, hence, do not exhibit branch cuts. Next, we use Newton's method to convert $u_{0}$ and $u_{N}$ into rational functions, so as to replace the branch cuts running from $\lambda=-k_{0}$ and $\lambda=-k_{N}$ with a set of pole singularities. This will make it possible to exactly evaluate the integrals through contour integration. According to Newton's method, the principal square root of the complex number $u_{n}^{2}$ (i.e., the principal root of the equation $\xi^{2}-u_{n}^{2}=0$ ) may be obtained by taking the limit of the sequence

$$
\tilde{u}_{n}^{(l)}=\frac{1}{2}\left[\tilde{u}_{n}^{(l-1)}+\frac{u_{n}^{2}}{\widetilde{u}_{n}^{(l-1)}}\right], \quad \tilde{u}_{n}^{(0)}=u_{n}^{2},
$$

as $l \rightarrow \infty$. Recursively applying (28) provides the explicit form of $\tilde{u}_{n}^{(l)}$; namely,

$$
\tilde{u}_{n}^{(l)}=\frac{2^{-l} P_{l}\left(u_{n}^{2}\right)}{\prod_{i=1}^{l-1} P_{i}\left(u_{n}^{2}\right)},
$$

with

$$
P_{i}(q)=\sum_{k=0}^{2^{i-1}}\left(\begin{array}{c}
2^{i} \\
2 k
\end{array}\right) q^{k}
$$

Thus, substitution of (29) for $u_{n}$ 's in (22) makes $\phi_{h}$ become a meromorphic function of $\lambda$, whose poles coincide with those of $\widetilde{u}_{0}^{(l)}$ and $\widetilde{u}_{N}^{(l)}$. The poles of $\widetilde{u}_{n}^{(l)}(n=0, N)$ with positive imaginary part, $M_{l}=2^{l-1}-1$ in number, are given by

$$
\lambda_{m n}^{(l)}=-\sqrt{p_{m}^{(l)}+k_{n}^{2}}
$$

with $p_{m}^{(l)}\left(m=1,2, \ldots, M_{l}\right)$ being the generic root of the equation

$$
\prod_{i=1}^{l-1} P_{i}(q)=0
$$

Notice that $2^{i-1}$ zeros of $P_{i}(q)$ are negative real numbers, and this implies that poles (31) are positioned on the suppressed branch lines, which are described by the equation

$$
\lambda^{2}-k_{n}^{2}=\alpha_{n}, \quad n=0, N,
$$

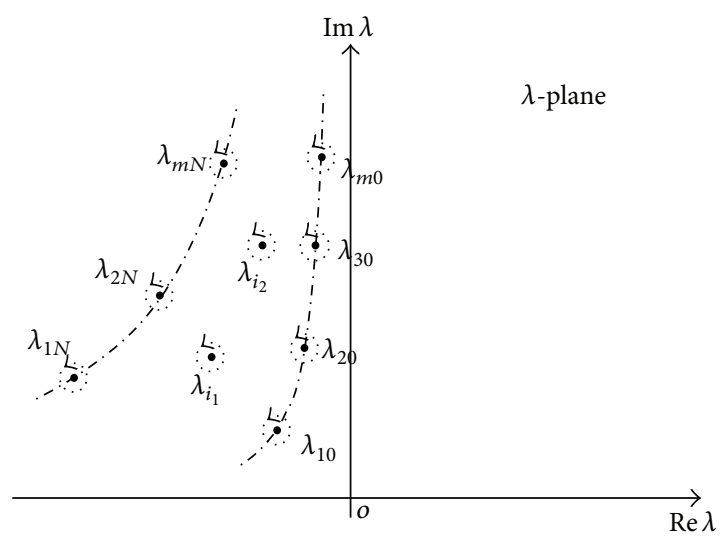

FIGURE 3: Final integration contour for the Sommerfeld Integrals.

with $\alpha_{n}$ being a negative real parameter. As shown in Figure 3, substitution (29) has the effect of turning the branch-cut integrals $S_{h}^{(0)}$ and $S_{h}^{(N)}$ into contour integrals around poles (31). It reads

$$
S_{h}^{(n)}=\sum_{m=1}^{M_{l}} \Phi_{h}^{(m n)}, \quad n=0, N
$$

where

$$
\begin{aligned}
\Phi_{h}^{(m 0)} & =\oint_{\Gamma_{m 0}}\left[\phi_{h}^{(o e)} \widetilde{u}_{0}+\phi_{h}^{(o o)} \widetilde{u}_{0} \widetilde{u}_{N}\right] H_{0}^{(1)}(\lambda \rho) \lambda d \lambda, \\
\Phi_{h}^{(m N)} & =\oint_{\Gamma_{m N}}\left[\phi_{h}^{(e o)} \tilde{u}_{N}+\phi_{h}^{(o o)} \tilde{u}_{0} \tilde{u}_{N}\right] H_{0}^{(1)}(\lambda \rho) \lambda d \lambda,
\end{aligned}
$$

with $\Gamma_{m n}(n=0, N)$ being the infinitesimal circle around $\lambda_{m n}$, and where the superscript $l$ has been omitted for notational simplicity. It should be observed that $\lambda_{m n}$ is not a pole of $\phi_{h}^{(e e)}$ and, hence, it results as

$$
\oint_{\Gamma_{m n}} \phi_{h}^{(e e)} H_{0}^{(1)}(\lambda \rho) \lambda d \lambda=0
$$

Substituting (29) for $\widetilde{u}_{0}$ and $\widetilde{u}_{N}$ in (35) provides the expressions

$$
\begin{aligned}
& \Phi_{h}^{(m 0)} \\
& \quad=\left\{\left[\phi_{h}^{(o e)}+\phi_{h}^{(o o)} u_{N}\right] H_{0}^{(1)}(\lambda \rho) \lambda\right\}_{\lambda=\lambda_{m 0}} \oint_{\Gamma_{m 0}} \widetilde{u}_{0} d \lambda, \\
& \Phi_{h}^{(m N)} \\
& \quad=\left\{\left[\phi_{h}^{(e o)}+\phi_{h}^{(o o)} u_{0}\right] H_{0}^{(1)}(\lambda \rho) \lambda\right\}_{\lambda=\lambda_{m N}} \oint_{\Gamma_{m N}} \tilde{u}_{N} d \lambda,
\end{aligned}
$$

where we have taken account of the fact that, far from the relevant poles, quantities $\tilde{u}_{0}$ and $\widetilde{u}_{N}$ may be confused with $u_{0}$ 
and $u_{N}$, respectively. Since from (6), (29), and (31) it follows that

$$
\begin{aligned}
\lim _{\lambda \rightarrow \lambda_{m n}} \tilde{u}_{n} & =2^{-l} P_{l}\left(p_{m}\right) R_{m} \lim _{\lambda \rightarrow \lambda_{m n}} \frac{1}{u_{n}^{2}-p_{m}} \\
& =2^{-l} P_{l}\left(p_{m}\right) R_{m} \lim _{\lambda \rightarrow \lambda_{m n}} \frac{1}{\lambda^{2}-\lambda_{m n}^{2}},
\end{aligned}
$$

with

$$
R_{m}=\prod_{\substack{m^{\prime}=1 \\ m^{\prime} \neq m}}^{M_{l}}\left[p_{m}-p_{m^{\prime}}\right]^{-1},
$$

applying Cauchy's integral formula [29] provides

$$
\begin{aligned}
\oint_{\Gamma_{m n}} \tilde{u}_{n} d \lambda & =2^{-l} P_{l}\left(p_{m}\right) R_{m} \oint_{\Gamma_{m n}} \frac{1}{\lambda^{2}-\lambda_{m n}^{2}} d \lambda \\
& =\frac{\pi j P_{l}\left(p_{m}\right) R_{m}}{2^{l} \lambda_{m n}} .
\end{aligned}
$$

Thus, (37) are turned into

$$
\begin{aligned}
\Phi_{h}^{(m 0)} & =\frac{\pi j P_{l}\left(p_{m}\right) R_{m} v_{h}\left(\lambda_{m 0}\right) H_{0}^{(1)}\left(\lambda_{m 0} \rho\right)}{2^{l}}, \\
\Phi_{h}^{(m N)} & =\frac{\pi j P_{l}\left(p_{m}\right) R_{m} v_{h}\left(\lambda_{m N}\right) H_{0}^{(1)}\left(\lambda_{m N} \rho\right)}{2^{l}},
\end{aligned}
$$

with

$$
v_{h}(\lambda)= \begin{cases}\phi_{h}^{(o e)}+\phi_{h}^{(o o)} u_{N}, & \lambda=\lambda_{m 0} \\ \phi_{h}^{(e o)}+\phi_{h}^{(o o)} u_{0}, & \lambda=\lambda_{m N} \\ 0, & \text { elsewhere }\end{cases}
$$

Combining (41) and (42) with (34) finally yields the expression

$$
\begin{aligned}
& S_{h}^{(0)}+S_{h}^{(N)}=\frac{\pi j}{2^{l}} \sum_{m=1}^{M_{l}} P_{l}\left(p_{m}\right) R_{m}\left[v_{h}\left(\lambda_{m 0}\right) H_{0}^{(1)}\left(\lambda_{m 0} \rho\right)\right. \\
& \left.+v_{h}\left(\lambda_{m N}\right) H_{0}^{(1)}\left(\lambda_{m N} \rho\right)\right]
\end{aligned}
$$

where one can clearly distinguish the above-surface ground wave, associated with $\lambda_{m 0}$ terms, and the lateral wave, constituted by $\lambda_{m N}$ terms, the latter traveling in the $N$ th layer of the medium along its upper boundary.

Substitution of (44) into (19), in conjunction with (21), provides explicit expressions, in series form, for integrals $S_{0}$ and $S_{1}$ appearing in (15)-(17).

\section{Validation of the Formulation}

To test the validity of the developed theory, formulas (15)-(17) and (19), (21), and (44) are applied to the computation of the

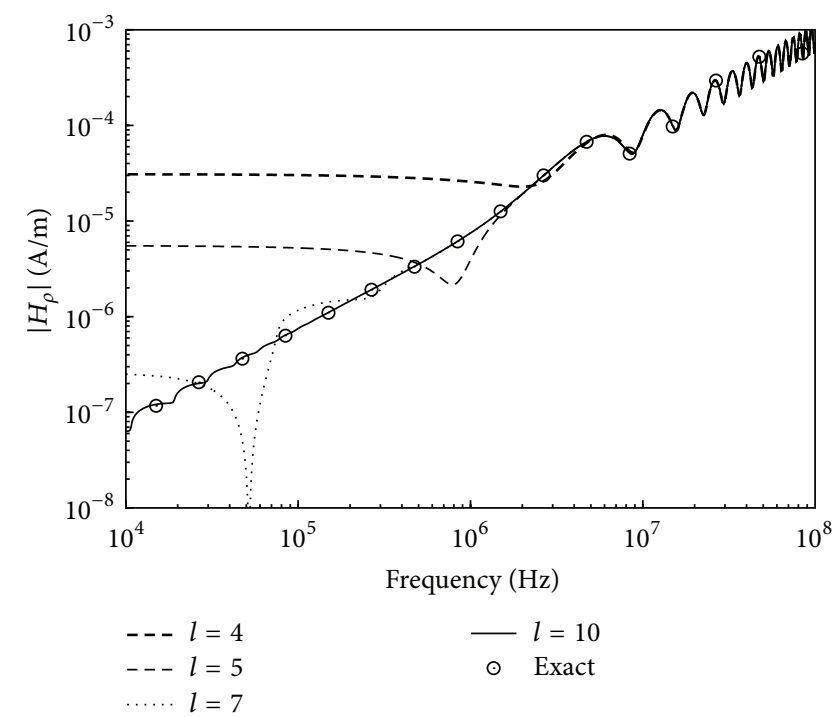

FIGURE 4: Amplitude-frequency spectrum of $H_{\rho}$, computed at $\rho=$ $20 \mathrm{~m}$ from the source point.

magnitude of the magnetic field components generated on the top surface of a homogeneous medium at distance $\rho$ from a unit-moment vertical magnetic dipole. In the considered case $N=1$, and functions $f_{h}(\lambda)$, which reduce to

$$
f_{h}(\lambda)=\frac{u_{0}^{h}}{u_{0}+u_{1}}
$$

do not exhibit poles. This implies that terms (21) do not contribute to the integrals. Thus, applying (24)-(26) provides

$$
\begin{aligned}
& \phi_{0}^{(e o)}=\phi_{1}^{(o o)}=-\phi_{0}^{(o e)}=\frac{1}{k_{0}^{2}-k_{1}^{2}}, \\
& \phi_{1}^{(e o)}=\phi_{1}^{(o e)}=\phi_{0}^{(o o)}=0,
\end{aligned}
$$

and integrals (19) assume the form

$$
\begin{aligned}
S_{0} & =\frac{\pi j}{2^{l}\left(k_{0}^{2}-k_{1}^{2}\right)} \sum_{m=1}^{M_{l}} P_{l}\left(p_{m}\right) R_{m}\left[H_{0}^{(1)}\left(\lambda_{m n} \rho\right)\right]_{n=0}^{n=1}, \\
S_{1} & =\frac{\pi j}{2^{l}\left(k_{0}^{2}-k_{1}^{2}\right)} \sum_{m=1}^{M_{l}} P_{l}\left(p_{m}\right) R_{m}\left[(-1)^{n}\right. \\
& \left.\cdot \sqrt{p_{m}+(-1)^{n}\left(k_{0}^{2}-k_{1}^{2}\right)} H_{0}^{(1)}\left(\lambda_{m n} \rho\right)\right]_{n=1}^{n=0} .
\end{aligned}
$$

The numerical results that arise from using (16) and (17), in combination with (47), are depicted in Figures 4-7 and denoted by lines. Such results are compared with those 


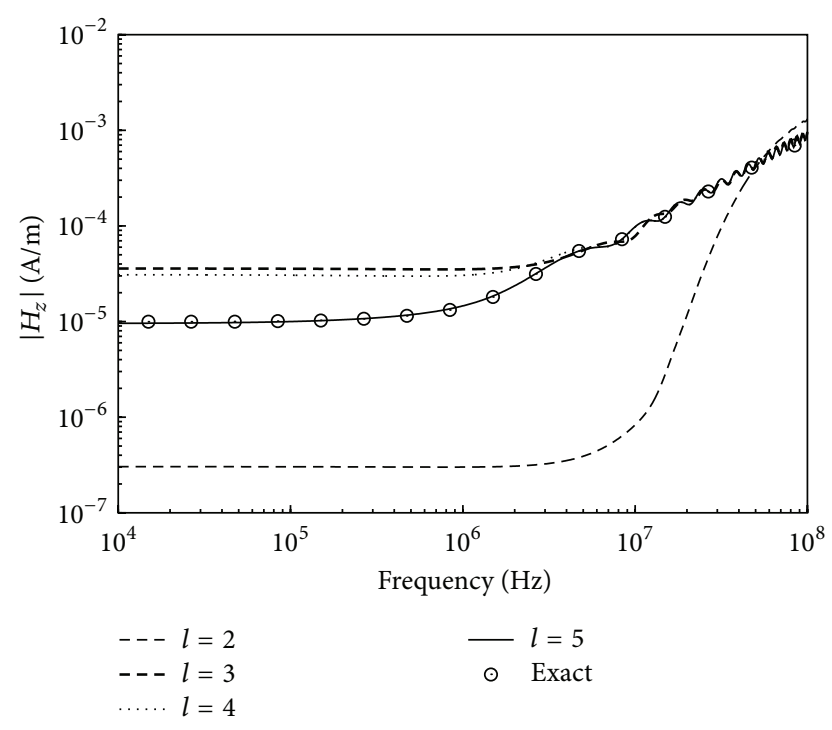

FIGURE 5: Amplitude-frequency spectrum of $H_{z}$, computed at $\rho=$ $20 \mathrm{~m}$ from the source point.

provided by the previously published exact expressions for $H_{\rho}$ and $H_{z}$; namely [16],

$$
\begin{aligned}
H_{\rho} & =\frac{m}{\pi \rho}\left[\frac{\alpha^{2}+\beta^{2}}{2} K_{1}(\alpha \rho) I_{1}(\beta \rho)\right. \\
& \left.-\alpha \beta K_{2}(\alpha \rho) I_{2}(\beta \rho)\right], \\
H_{z} & =\frac{Q_{1}-Q_{0}}{2 \pi\left(k_{0}^{2}-k_{1}^{2}\right)},
\end{aligned}
$$

where $I_{n}(\cdot)$ and $K_{n}(\cdot)$ are the $n$ th-order modified Bessel functions of the first and second kind, respectively, and

$$
\begin{aligned}
& \alpha=\frac{1}{2} j\left(k_{1}+k_{0}\right), \\
& \beta=\frac{1}{2} j\left(k_{1}-k_{0}\right),
\end{aligned}
$$

with

$$
Q_{n}=\left(-j k_{n}^{3} \rho^{3}-4 k_{n}^{2} \rho^{2}+9 j k_{n} \rho+9\right) \frac{e^{-j k_{n} \rho}}{\rho^{5}} .
$$

Figures 4 and 5 present amplitude-frequency spectra of $H_{\rho}$ - and $H_{z}$-fields generated at $\rho=20 \mathrm{~m}$, obtained assuming $\sigma_{1}=1 \mathrm{mS} / \mathrm{m}$ and $\epsilon_{1}=10 \epsilon_{0}$. Each figure illustrates the behavior of the sequence of spectra generated by the proposed solution as the number of iterations of Newton's method $(l)$ is increased. As is seen, the sequences converge, and the number of iterations required to achieve perfect agreement with the exact data, denoted by points, is 10 for $H_{\rho}$-component and 5 for $H_{z}$-component. This means that the sums $S_{1}$ and $S_{0}$ in (47) must be made up of $M_{10}=2^{9}-1=511$ terms and $M_{5}=2^{4}-1=15$ terms, respectively.

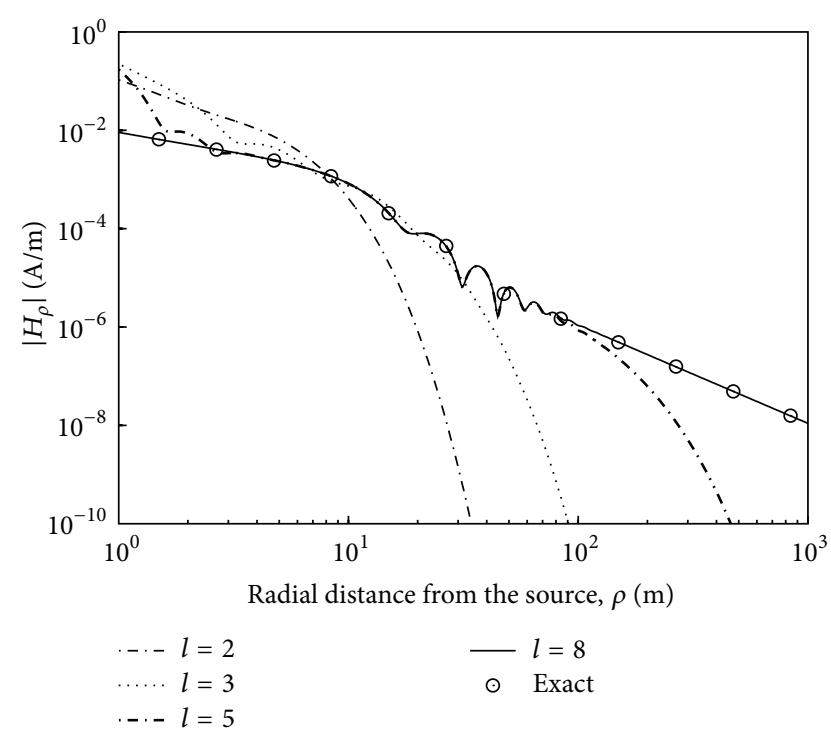

FIGURE 6: Profiles of $\left|H_{\rho}\right|$ against the radial distance from the source point.

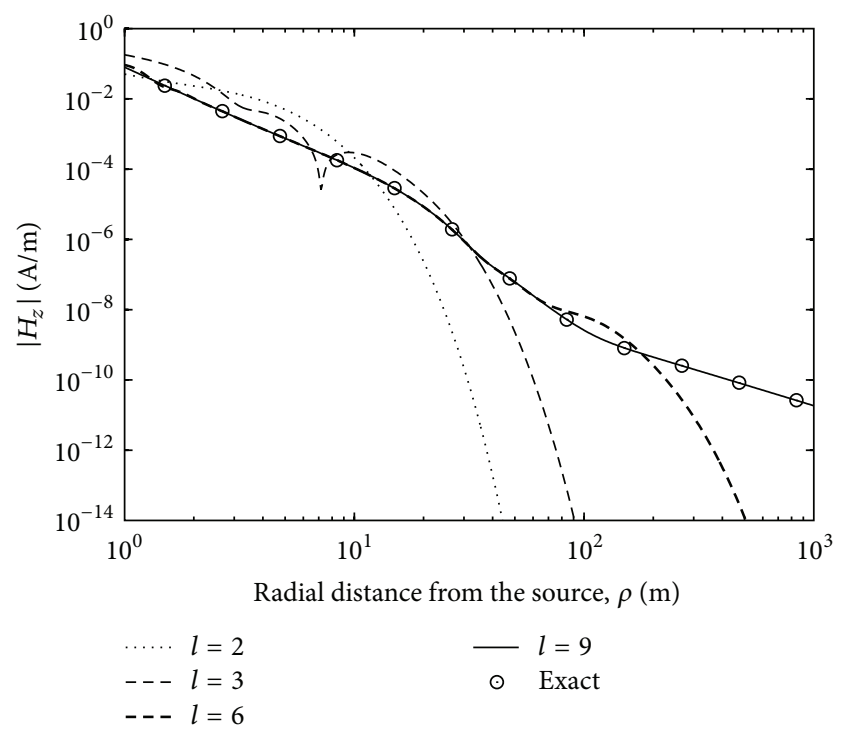

Figure 7: Profiles of $\left|H_{z}\right|$ against the radial distance from the source point.

It should be observed that the dominant contributions to the sums in (47) come from the residues at the poles located in the portions of the branch cuts which are closer to the real axis, since the magnitude of $H_{0}^{(1)}(\lambda \rho)$ is significantly larger at them. In particular, the ground-wave poles $\left(\lambda_{m 0}\right.$ 's $)$ that lie on the negative real $\lambda$-axis contribute most to $S_{0}$ and $S_{1}$. For fixed $l$, the number of such poles diminishes as frequency is decreased, and this happens because the branch point $-k_{0}$ gets closer to the origin of the complex plane. The consequence of this phenomenon is that, at low frequencies, the number of dominant residues may not be sufficient to achieve accurate results. This is the reason why, as illustrated in Figure 4, accurate evaluation of $H_{\rho}$ requires 15 terms if 
frequency is higher than $10 \mathrm{MHz}$ and at least 511 terms when frequency is lower than $100 \mathrm{kHz}$.

Notice that the onset of oscillations in the frequency spectra is expected, since, when entering the far zone, the total field results from the superposition of two waves, which propagate with wavenumbers $k_{0}$ and $k_{1}$ and decay with distance $\rho$ from the source point. Physically, this means that oscillatory interference patterns arise in both space and frequency domains. In the space domain, the period of the oscillations is $2 \pi /\left(k_{1}-k_{0}\right)$, with $k_{1}$ approaching a real quantity since, at high frequencies, the displacement currents predominate over the conduction currents. In the frequency domain, the patterns oscillate with period

$$
\Lambda=\frac{1}{\rho \sqrt{\mu_{0} \epsilon_{0}}\left(\sqrt{\epsilon_{r_{1}}}-1\right)},
$$

which, for $\rho=20 \mathrm{~m}$ and $\epsilon_{r_{1}}=10$, is equal to

$$
\Lambda=\frac{3 \cdot 10^{8}}{20(\sqrt{10}-1)} \cong 6.94 \mathrm{MHz} \text {. }
$$

It may be numerically verified that 6.94 is just the peak-topeak spacing of the far-zone asymptotic trend of each solidline curve in Figures 4 and 5.

Finally, Figures 6 and 7 show profiles of the amplitudes of $H_{\rho}$ and $H_{z}$ against the source-receiver distance $\rho$. The calculations have been performed assuming that the loop antenna is positioned on the same medium as in the previous examples and that it operates at $10 \mathrm{MHz}$. The plotted curves demonstrate that even when taken as functions of $\rho$, the sequences of partial sums in (47) are convergent and give rise to accurate results after only $8\left(H_{\rho}\right.$-field $)$ and $9\left(H_{z}\right.$-field $)$ iterations of Newton's method.

\section{Conclusion}

This paper has presented exact series representations for the surface fields excited by a small circular loop antenna placed on the top surface of plane stratified earth. The derived expressions result from a rigorous analytical procedure, which allows casting the complete integral representations for the EM field components into forms suitable for application of Cauchy's integral formula. Numerical results are presented to show the validity and accuracy of the proposed formulation.

\section{Conflict of Interests}

The author declares that there is no conflict of interests regarding the publication of this paper.

\section{References}

[1] L. B. Felsen and N. Marcuvitz, Radiation and Scattering of Waves, IEEE Press, Piscataway, NJ, USA, 1994.

[2] V. I. Okhmatovski and A. C. Cangellaris, "Evaluation of layered media green's functions via rational function fitting," IEEE Microwave and Wireless Components Letters, vol. 14, no. 1, pp. 22-24, 2004.
[3] M. Parise, "A study on energetic efficiency of coil antennas used for RF diathermy," IEEE Antennas and Wireless Propagation Letters, vol. 10, pp. 385-388, 2011.

[4] Z. H. Firouzeh, G. A. E. Vandenbosch, R. Moini, S. H. H. Sadeghi, and R. Faraji-Dana, "Efficient evaluation of green's functions for lossy half-space problems," Progress in Electromagnetics Research, vol. 109, pp. 139-157, 2010.

[5] L. M. Brekhovskikh, Waves in Layered Media, Academic Press, New York, NY, USA, 1960.

[6] L. B. Proekt and A. Cangellaris, "An approximation of the electromagnetic Green's function of layered media with the source point considered as an independent variable," IEEE Transactions on Magnetics, vol. 40, no. 2, pp. 1037-1040, 2004.

[7] A. Baños, Dipole Radiation in the Presence of a Conducting HalfSpace, Pergamon Press, Oxford, NY, USA, 1966.

[8] R. K. Moore and W. E. Blair, "Dipole radiation in a conducting half space," Journal of Research of the National Bureau of Standards Section D: Radio Propagation, vol. 65, no. 6, pp. 547563, 1961.

[9] M. Parise, "On the use of cloverleaf coils to induce therapeutic heating in tissues," Journal of Electromagnetic Waves and Applications, vol. 25, no. 11-12, pp. 1667-1677, 2011.

[10] J. R. Wait, "The electromagnetic fields of a horizontal dipole in the presence of a conducting half-space," Canadian Journal of Physics, vol. 39, no. 7, pp. 1017-1028, 1961.

[11] J. A. Kong, Electromagnetic Wave Theory, John Wiley \& Sons, New York, NY, USA, 1986.

[12] S. H. Ward and G. W. Hohmann, "Electromagnetic theory for geophysical applications," in Electromagnetic Methods in Applied Geophysics, vol. 1, chapter 4, pp. 130-311, Society of Exploration Geophysicists, Tulsa, Okla, USA, 1988.

[13] J. R. Wait, Electromagnetic Waves in Stratified Media, Pergamon Press, New York, NY, USA, 1970.

[14] G. J. Palacky, "Resistivity characteristics of geologic targets," in Electromagnetic Methods in Applied Geophysics, M. N. Nabighian, Ed., vol. 1, chapter 3, pp. 52-129, Society of Exploration Geophysicists, Tulsa, Okla, USA, 1988.

[15] M. Parise, "Fast computation of the forward solution in controlled-source electromagnetic sounding problems," Progress in Electromagnetics Research, vol. 111, pp. 119-139, 2011.

[16] M. Parise, "Exact electromagnetic field excited by a vertical magnetic dipole on the surface of a lossy half-space," Progress in Electromagnetics Research B, vol. 23, pp. 69-82, 2010.

[17] J. R. Wait, "Influence of a sub-surface insulating layer on electromagnetic ground wave propagation," IEEE Transactions on Antennas and Propagation, vol. 14, no. 6, pp. 755-767, 1966.

[18] W. C. Chew, Waves and Fields in Inhomogeneous Media, IEEE Press, New York, NY, USA, 1995.

[19] A. Arutaki and J. Chiba, "Communication in a three-layered conducting media with a vertical magnetic dipole," IEEE Transactions on Antennas and Propagation, vol. 28, no. 4, pp. 551-556, 1980.

[20] P. R. Bannister, "The quasi-static fields of a horizontal magnetic dipole," Tech. Rep. 698, Navy Underwater Sound Laboratory, New London, Conn, USA, 1965.

[21] R. W. P. King, "Electromagnetic field of a vertical dipole over an imperfectly conducting half-space," Radio Science, vol. 25, no. 2, pp. 149-160, 1990.

[22] D. Guptasarma and B. Singh, "New digital linear filters for Hankel $J_{0}$ and $J_{1}$ transforms," Geophysical Prospecting, vol. 45, no. 5, pp. 745-762, 1997. 
[23] N. P. Singh and T. Mogi, "Electromagnetic response of a large circular loop source on a layered Earth: a new computation method," Pure and Applied Geophysics, vol. 162, no. 1, pp. 181200, 2005.

[24] M. Parise and S. Cristina, "High-order electromagnetic modeling of shortwave inductive diathermy effects," Progress in Electromagnetics Research, vol. 92, pp. 235-253, 2009.

[25] F. N. Kong, "Hankel transform filters for dipole antenna radiation in a conductive medium," Geophysical Prospecting, vol. 55, no. 1, pp. 83-89, 2007.

[26] T. J. McDougall and S. J. Wotherspoon, "A simple modification of Newton's method to achieve convergence of order $1+\sqrt{2}$," Applied Mathematics Letters, vol. 29, pp. 20-25, 2014.

[27] S. Ilic, M. Petkovic, and D. Herceg, "A note on babylonian square-root algorithm and related variants," Novi Sad Journal of Mathematics, vol. 26, pp. 155-162, 1996.

[28] I. S. Gradshteyn and I. M. Ryzhik, Table of Integrals, Series, and Products, edited by A. Jeffrey and D. Zwillinger, Academic Press, New York, NY, USA, 2007.

[29] E. T. Whittaker and G. N. Watson, A Course of Modern Analysis, Cambridge University Press, Cambridge, UK, 4th edition, 1996. 

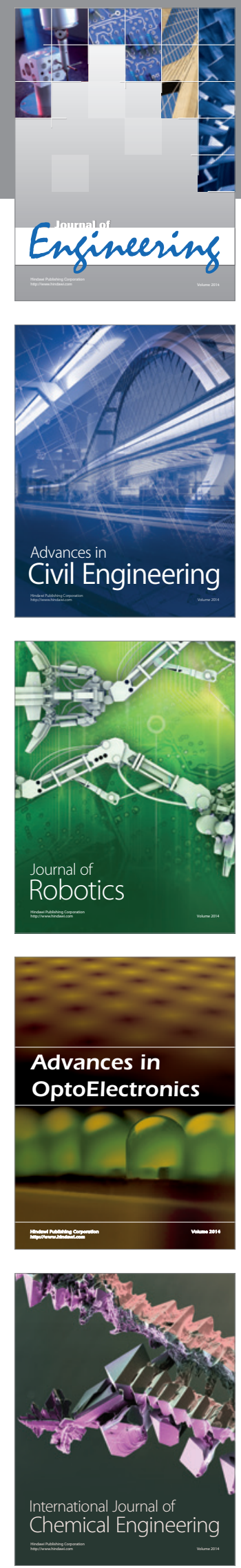

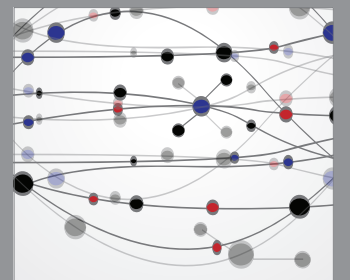

The Scientific World Journal
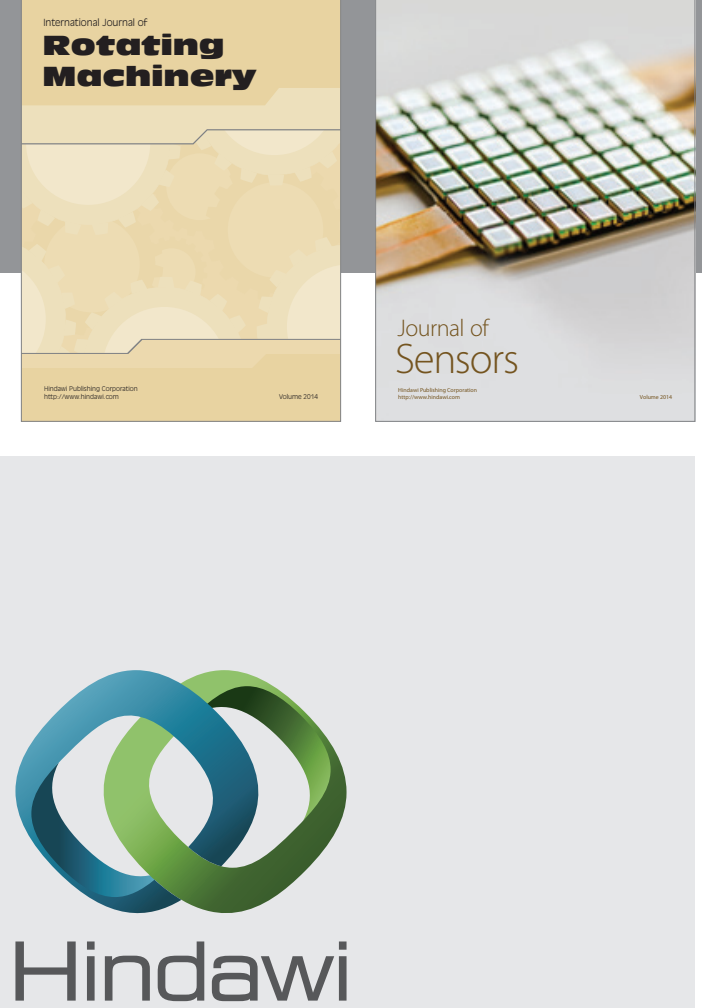

Submit your manuscripts at http://www.hindawi.com
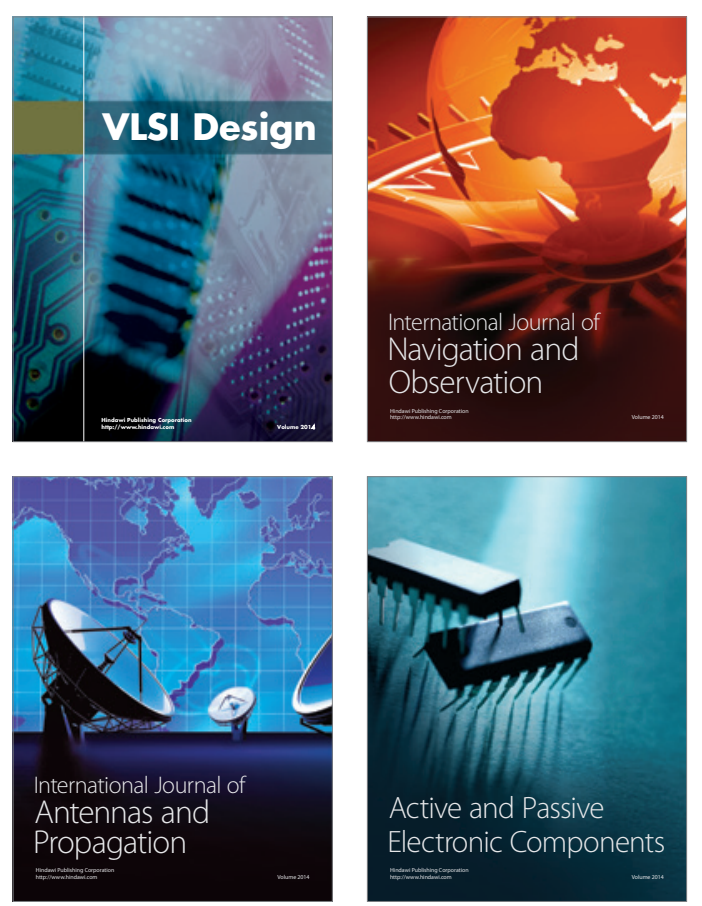
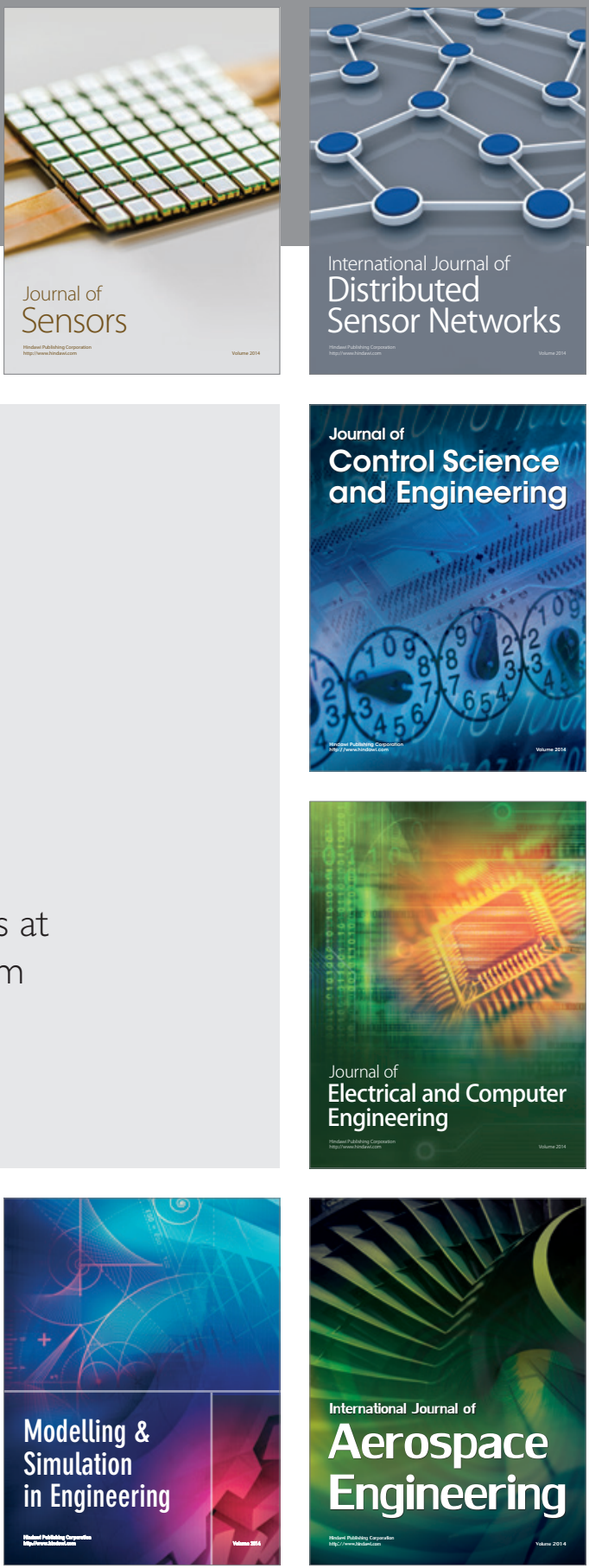

Journal of

Control Science

and Engineering
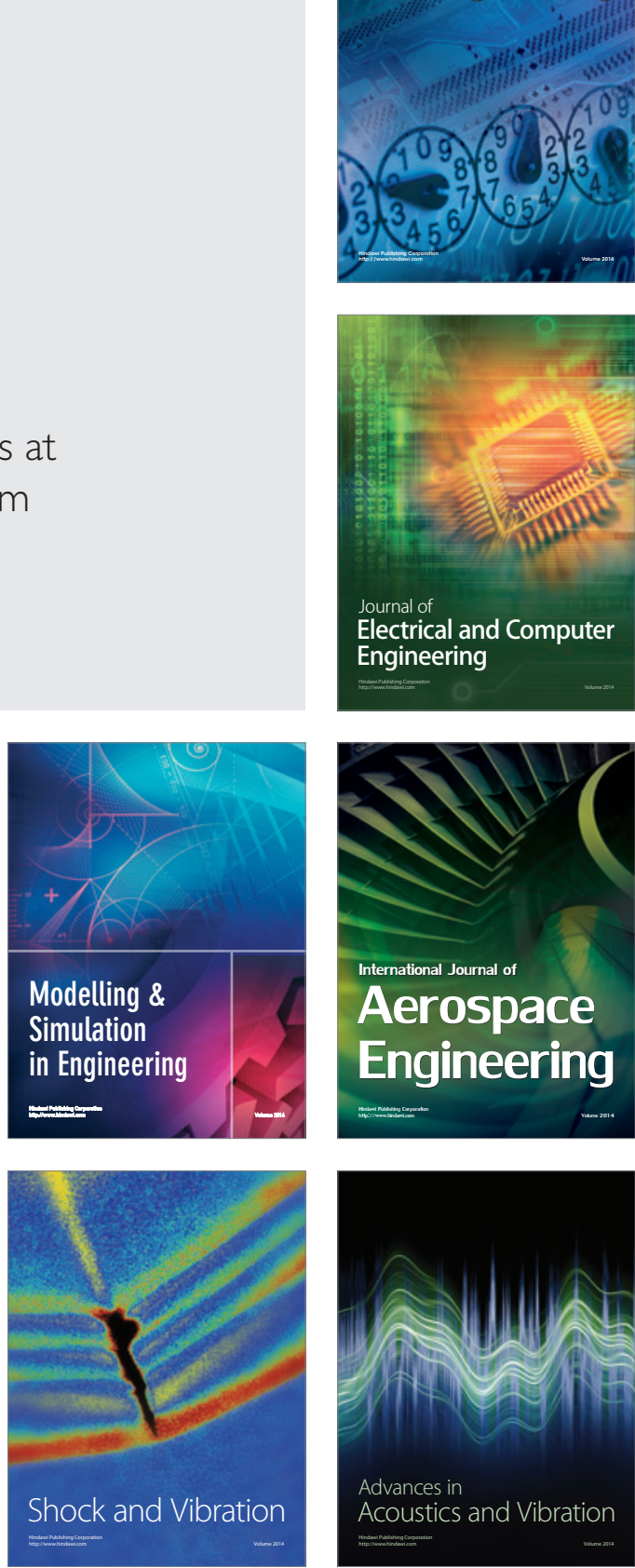